\title{
Human bladder cancer risk calculation based on genome-wide analysis of genetic variants
}

\author{
H. M. Bolt
}

Published online: 13 February 2013

(C) Springer-Verlag Berlin Heidelberg 2013

Urinary bladder cancer is the ninth most common cancer worldwide (Roth et al. 2012; Golka et al. 2011). The strongest known risk factors are cigarette smoking, occupational exposure to bladder carcinogens and male gender (Golka et al. 2012a, b; Ovsiannikov et al. 2012). It is well established that slow acetylation (NAT2) and a deletion variant of glutathione S-transferase M1 (GSTM1) are associated with increased bladder cancer risk (Schwender et al. 2012; Bell et al. 1993; Cartwright et al. 1984; Golka et al. 1996; Kempkes et al. 1996; Hengstler et al. 1998). Recently, genome-wide association studies have identified further polymorphisms, and their influence was confirmed in independent case-control series (Kiemeney et al. 2008, 2010; Rothman et al. 2010; Rafnar et al. 2009, 2011; Wu et al. 2009; Golka et al. 2009; Lehmann et al. 2010; Selinski et al. 2011; Binder et al. 2012; Bolt 2012). All the information currently known for the 13 polymorphisms has recently been summarized (Selinski 2012). Although it is highly likely that the most influential individual polymorphisms have already been identified, it remains unknown whether these polymorphism interact with one another leading to a higher risk of bladder cancer, in the case where an individual carries several high-risk alleles.

In the current issue of PLOS one, Holger Schwender and colleagues from TU Dortmund University established a technique to calculate whether there is interaction among the high-risk alleles and how this influences bladder cancer risk (Schwender et al. 2012). The authors used six of the

H. M. Bolt $(\square)$

Leibniz Institut für Arbeitsforschung an der TU Dortmund, Leibniz Research Centre for Working Environment and Human Factors (IfADo), Ardeystrasse 67, 44139 Dortmund, Germany e-mail: bolt@ifado.de previously identified single nucleotide polymorphisms (SNPs) in a bladder cancer case-control series of 1,595 bladder cancer cases and 1,760 controls. The authors tested all possible SNP combinations and their stability by using a bootstrap technique. The take home messages of this study are as follows:

- Certain high-risk alleles interact; however, their interaction is less than additive.

- Different SNP combinations are relevant in smokers and non-smokers.

- The top scoring 'smoker variants' include variants of GSTM1 and UGT1A, both involved in detoxification of cigarette-smoke carcinogens.

- The top scoring 'non-smoker variants' include APOBEC3A and cMYC, both involved in the proliferation and maintenance of DNA integrity.

- The highest stable combination effect results in an odds ratio of approximately 2.0 (combined influence of the high-risk alleles of rs 9642880, rs 710521 and rs 1014971 in non-smokers).

- However, the degree to which genetic disposition contributes to cancer risk still seems to be smaller than that of environmental factors. The odds ratio of cigarette smoking is approximately 3.5 and is therefore still higher than the combined influence of the high-risk alleles.

The study of Schwender et al. represents an important milestone on the path to calculate the cancer risk associated with entire genomes. A limitation of the current study is the relatively low number of cases $(1,595)$, which will at most allow the analysis of three-way interactions. Therefore, an important next step in this field of research will be to conduct a SNP interaction study that includes all cell cases and controls available worldwide. 


\section{References}

Bell DA, Taylor JA, Paulson DF, Robertson CN, Mohler JL, Lucier GW (1993) Genetic risk and carcinogen exposure: a common inherited defect of the carcinogen-metabolism gene glutathione S-transferase M1 (GSTM1) that increases susceptibility to bladder cancer. J Natl Cancer Inst 85:1159-1164

Binder H, Müller T, Schwender H, Golka K, Steffens M, Hengstler JG, Ickstadt K, Schumacher M (2012) Cluster-localized sparse logistic regression for SNP data. Stat Appl Genet Mol Biol 11(4). doi:10.1515/1544-6115.1694

Bolt HM (2012) Relevance of genetic disposition versus environmental exposure for cancer risk: an old controversy revisited with novel methods. EXCLI J 12:79-80

Cartwright RA, Glashan RW, Rogers HJ, Ahmad RA, Barham-Hall D et al (1984) Role of N-acetyltransferase phenotypes in bladder carcinogenesis: a pharmacogenetic epidemiological approach to bladder cancer. Lancet 2:842-845

Golka K, Prior V, Blaszkewicz M, Cascorbi I, Schöps W et al (1996) Occupational history and genetic $\mathrm{N}$-acetyltransferase polymorphism in urothelial cancer patients of Leverkusen, Germany. Scand J Work Environ Health 22:332-338

Golka K, Hermes M, Selinski S, Blaszkewicz M, Bolt HM, Roth G, Dietrich H, Prager HM, Ickstadt K, Hengstler JG (2009) Susceptibility to urinary bladder cancer: relevance of rs9642880[T], GSTM1 0/0 and occupational exposure. Pharmacogenet Genomics 19(11):903-906

Golka K, Selinski S, Lehmann ML, Blaszkewicz M, Marchan R, Ickstadt K, Schwender H, Bolt HM, Hengstler JG (2011) Genetic variants in urinary bladder cancer: collective power of the "wimp SNPs". Arch Toxicol 85(6):539-554

Golka K, Abreu-Villaca Y, Anbari Attar R, Angeli-Greaves M, Aslam M, Basaran N, Belik R, Butryee C, Dalpiaz O, Dzhusupov K, Ecke TH, Galambos H, Galambos H, Gerilovica H, Gerullis H, Gonzalez PC, Goossens ME, Gorgishvili-Hermes L, Heyns CF, Hodzic J, Ikoma F, Jichlinski P, Kang BH, Kiesswetter E, Krishnamurthi K, Lehmann ML, Martinova I, Mittal RD, Ravichandran B, Romics I, Roy B, Rungkat-Zakaria F, Rydzynski K, Scutaru C, Shen J, Soufi M, Toguzbaeva K, Vu Duc T, Widera A, Wishahi M, Hengstler JG (2012a) Bladder cancer documentation of causes: multilingual questionnaire, 'bladder cancer doc'. Front Biosci (Elite Ed) 4:2809-2822

Golka K, Kopps S, Prager HM, Mende Sv, Thiel R, Jungmann O, Zumbe J, Bolt HM, Blaszkewicz M, Hengstler JG, Selinski S (2012b) Bladder cancer in crack testers applying azo dye-based sprays to metal bodies. J Toxicol Environ Health A 75(8-10):566-571

Hengstler JG, Arand M, Herrero ME, Oesch F (1998) Polymorphisms of $\mathrm{N}$-acetyltransferases, glutathione S-transferases, microsomal epoxide hydrolase and sulfotransferases: influence on cancer susceptibility. Recent Results Cancer Res 154:47-85

Kempkes M, Golka K, Reich S, Reckwitz T, Bolt HM (1996) Glutathione S-transferase GSTM1 and GSTT1 null genotypes as potential risk factors for urothelial cancer of the bladder. Arch Toxicol 71:123-126

Kiemeney LA, Thorlacius S, Sulem P, Geller F, Aben KK, Stacey SN, Gudmundsson J, Jakobsdottir M, Bergthorsson JT, Sigurdsson A, Blondal T, Witjes JA, Vermeulen SH, Hulsbergenvan de Kaa CA, Swinkels DW, Ploeg M, Cornel EB, Vergunst $\mathrm{H}$, Thorgeirsson TE, Gudbjartsson D, Gudjonsson SA, Thorleifsson G, Kristinsson KT, Mouy M, Snorradottir S, Placidi D, Campagna M, Arici C, Koppova K, Gurzau E, Rudnai P, Kellen E, Polidoro S, Guarrera S, Sacerdote C, Sanchez M, Saez B, Valdivia G, Ryk C, de Verdier P, Lindblom A, Golka K, Bishop DT, Knowles MA, Nikulasson S, Petursdottir V,
Jonsson E, Geirsson G, Kristjansson B, Mayordomo JI, Steineck G, Porru S, Buntinx F, Zeegers MP, Fletcher T, Kumar R, Matullo G, Vineis P, Kiltie AE, Gulcher JR, Thorsteinsdottir U, Kong A, Rafnar T, Stefansson K (2008) Sequence variant on $8 \mathrm{q} 24$ confers susceptibility to urinary bladder cancer. Nat Genet 40(11):1307-1312

Kiemeney LA, Sulem P, Besenbacher S, Vermeulen SH, Sigurdsson A, Thorleifsson G, Gudbjartsson DF, Stacey SN, Gudmundsson J, Zanon C, Kostic J, Masson G, Bjarnason H, Palsson ST, Skarphedinsson OB, Gudjonsson SA, Witjes JA, Grotenhuis AJ, Verhaegh GW, Bishop DT, Sak SC, Choudhury A, Elliott F, Barrett JH, Hurst CD, de Verdier PJ, Ryk C, Rudnai P, Gurzau E, Koppova K, Vineis P, Polidoro S, Guarrera S, Sacerdote C, Campagna M, Placidi D, Arici C, Zeegers MP, Kellen E, Gutierrez BS, Sanz-Velez JI, Sanchez-Zalabardo M, Valdivia G, Garcia-Prats MD, Hengstler JG, Blaszkewicz M, Dietrich H, Ophoff RA, van den Berg LH, Alexiusdottir K, Kristjansson K, Geirsson G, Nikulasson S, Petursdottir V, Kong A, Thorgeirsson T, Mungan NA, Lindblom A, van Es MA, Porru S, Buntinx F, Golka K, Mayordomo JI, Kumar R, Matullo G, Steineck G, Kiltie AE, Aben KK, Jonsson E, Thorsteinsdottir U, Knowles MA, Rafnar T, Stefansson K (2010) A sequence variant at 4 p16.3 confers susceptibility to urinary bladder cancer. Nat Genet 42(5):415-419

Lehmann ML, Selinski S, Blaszkewicz M, Orlich M, Ovsiannikov D, Moormann O, Guballa C, Kress A, Truss MC, Gerullis H, Otto T, Barski D, Niegisch G, Albers P, Frees S, Brenner W, Thüroff JW, Angeli-Greaves M, Seidel T, Roth G, Dietrich H, Ebbinghaus R, Prager HM, Bolt HM, Falkenstein M, Zimmermann A, Klein T, Reckwitz T, Roemer HC, Löhlein D, Weistenhöfer W, Schöps W, Beg AE, Aslam M, Bánfi G, Romics I, Ickstadt K, Schwender H, Winterpacht A, Hengstler JG, Golka K (2010) Rs710521[A] on chromosome $3 \mathrm{q} 28$ close to TP63 is associated with increased urinary bladder cancer risk. Arch Toxicol 84(12):967-978

Ovsiannikov D, Selinski S, Lehmann ML, Blaszkewicz M, Moormann O, Haenel MW, Hengstler JG, Golka K (2012) Polymorphic enzymes, urinary bladder cancer risk, and structural change in the local industry. $\mathrm{J}$ Toxicol Environ Health A 75(8-10):557-565

Rafnar T, Sulem P, Stacey SN, Geller F, Gudmundsson J et al (2009) Sequence variants at the TERT-CLPTM1L locus associate with many cancer types. Nat Genet 41:221-227

Rafnar T, Vermeulen SH, Sulem P, Thorleifsson G, Aben KK, Witjes JA, Grotenhuis AJ, Verhaegh GW, Hulsbergen-van de Kaa CA, Besenbacher S, Gudbjartsson D, Stacey SN, Gudmundsson J, Johannsdottir H, Bjarnason $\mathrm{H}$, Zanon C, Helgadottir H, Jonasson JG, Tryggvadottir L, Jonsson E, Geirsson G, Nikulasson S, Petursdottir V, Bishop DT, Chung-Sak S, Choudhury A, Elliott F, Barrett JH, Knowles MA, de Verdier PJ, Ryk C, Lindblom A, Rudnai P, Gurzau E, Koppova K, Vineis P, Polidoro S, Guarrera S, Sacerdote C, Panadero A, Sanz-Velez JI, Sanchez M, Valdivia G, Garcia-Prats MD, Hengstler JG, Selinski S, Gerullis H, Ovsiannikov D, Khezri A, Aminsharifi A, Malekzadeh M, van den Berg LH, Ophoff RA, Veldink JH, Zeegers MP, Kellen E, Fostinelli J, Andreoli D, Arici C, Porru S, Buntinx F, Ghaderi A, Golka K, Mayordomo JI, Matullo G, Kumar R, Steineck G, Kiltie AE, Kong A, Thorsteinsdottir U, Stefansson K, Kiemeney LA (2011) European genome-wide association study identifies SLC14A1 as a new urinary bladder cancer susceptibility gene. Hum Mol Genet 20(21):4268-4281

Roth E, Selinski S, Schikowsky C, Seidel T, Volkert F, Blaszkewicz M, Hengstler JG, Golka K (2012) Bladder cancer survival in a former industrial area in Saxony-Anhalt, Germany. J Toxicol Environ Health A 75(19-20):1216-1225 
Rothman N, Garcia-Closas M, Chatterjee N, Malats N, Wu X, Figueroa JD, Real FX, Van Den Berg D, Matullo G, Baris D, Thun M, Kiemeney LA, Vineis P, De Vivo I, Albanes D, Purdue MP, Rafnar T, Hildebrandt MA, Kiltie AE, Cussenot O, Golka K, Kumar R, Taylor JA, Mayordomo JI, Jacobs KB, Kogevinas M, Hutchinson A, Wang Z, Fu YP, Prokunina-Olsson L, Burdett L, Yeager M, Wheeler W, Tardón A, Serra C, Carrato A, GarcíaClosas R, Lloreta J, Johnson A, Schwenn M, Karagas MR, Schned A, Andriole G Jr, Grubb R III, Black A, Jacobs EJ, Diver WR, Gapstur SM, Weinstein SJ, Virtamo J, Cortessis VK, GagoDominguez M, Pike MC, Stern MC, Yuan JM, Hunter DJ, McGrath M, Dinney CP, Czerniak B, Chen M, Yang H, Vermeulen SH, Aben KK, Witjes JA, Makkinje RR, Sulem P, Besenbacher S, Stefansson K, Riboli E, Brennan P, Panico S, Navarro C, Allen NE, Bueno-de-Mesquita HB, Trichopoulos D, Caporaso N, Landi MT, Canzian F, Ljungberg B, Tjonneland A, Clavel-Chapelon F, Bishop DT, Teo MT, Knowles MA, Guarrera S, Polidoro S, Ricceri F, Sacerdote C, Allione A, Cancel-Tassin G, Selinski S, Hengstler JG, Dietrich H, Fletcher T, Rudnai P, Gurzau E, Koppova K, Bolick SC, Godfrey A, Xu Z, Sanz-Velez JI D, García-Prats M, Sanchez M, Valdivia G, Porru S, Benhamou S, Hoover RN, Fraumeni JF Jr, Silverman DT, Chanock SJ (2010) A multi-stage genome-wide association study of bladder cancer identifies multiple susceptibility loci. Nat Genet 42(11):978-984
Schwender H, Selinski S, Blaszkewicz M, Marchan R, Ickstadt K, Golka K, Hengstler JG (2012) Distinct SNP combinations confer susceptibility to urinary bladder cancer in smokers and nonsmokers. PLoS One 7(12):51880. doi:10.1371/journal.pone. 0051880

Selinski S (2012) Genetic variants confer susceptibility to urinary bladder cancer: an updated list of confirmed polymorphisms. EXCLI J 11:743-747

Selinski S, Blaszkewicz M, Lehmann ML, Ovsiannikov D, Moormann O, Guballa C, Kress A, Truss MC, Gerullis H, Otto T, Barski D, Niegisch G, Albers P, Frees S, Brenner W, Thüroff JW, Angeli-Greaves M, Seidel T, Roth G, Dietrich H, Ebbinghaus R, Prager HM, Bolt HM, Falkenstein M, Zimmermann A, Klein T, Reckwitz T, Roemer HC, Löhlein D, Weistenhöfer W, Schöps W, Hassan Rizvi SA, Aslam M, Bánfi G, Romics I, Steffens M, Ekici AB, Winterpacht A, Ickstadt K, Schwender H, Hengstler JG, Golka K (2011) Genotyping NAT2 with only two SNPs (rs1041983 and rs1801280) outperforms the tagging SNP rs1495741 and is equivalent to the conventional 7-SNP NAT2 genotype. Pharmacogenet Genomics 21(10):673-678

Wu X, Ye Y, Kiemeney LA, Sulem P, Rafnar T et al (2009) Genetic variation in the prostate stem cell antigen gene PSCA confers susceptibility to urinary bladder cancer. Nat Genet 41:991-995 [Erratum in: Nat Genet 41: 1156] 